\title{
Die Amphibienfauna von Neu-Guinea
}

\author{
VON
}

\author{
P. N. VAN KAMPEN.
}

Unsere Kenntnis der Fauna von Neu-Guinea, welche längere Zeit ausschliesslich auf die Küstengegenden beschränkt geblieben war, ha乇 infolge der zahlreichen Forschungsreisen der letzten Dezennien in dem Masse zuggenommen, dass man jetzt wohl annehmen darf, dass sie uns, wenigstens was die Vertebraten anbelangt, der Hauptsache nach bekannt ist.

Als ein Beispiel der schnellen Vermehrung dieser Kenntnis sind die Amphibien zu nennen. Noch im Jahre 1882 waren, wie aus Boulenger's Katalog des „British Museum” hervorgeht, nur 17 Arten dieser Klasse von Neu-Guinea bekannt; aus der unten folgenden Aufzählung ist ersichtlich, dass diese Zahl zurzeit um mehr als das vierfache zugenommen hat. Besonders die Durchforschung des zentralen Gebirges hat dazu beigetragen.

Bei der ziemlich zerstreuten Literatur wird es seinen Nützen haben, eine kritische Übersicht der jetzt von Neu-Guinea bekannten Amphibien zu geben und einige zoogeographische Betrachtungen damit zu verknüpfen. Es freut mich diese kleine. Zusammenfassung Herrn Dr. Kerbert widmen zu können, dem Director der Zoologischen Gesellschaft „Natura Artis Magistra", welche sich durch ihre zoologische Sammlungen und durch Unterstützung von wissenschaftlichen Reisen grosse Verdienste um die faunistische Untersuchung des Indischen Archipels erworben hat.

Die Amphibien von Neu-Guinea gehören zu den Familien der Pelobatidae, Hylidae, Cystignathidae, Ranidae und Engystomatidae, von welchen die zweite und fünfte bei weitem am zahlreichsten vertreten sind. Auf einigen der benachbarten kleineren Inseln des papuanischen Gebietes leben ausserdem die einzigen Vertreter der Familien, oder vielleicht besser Subfamilien der Ceratobatrachidae (Ceratobatrachus Guentheri Blgr. auf den. Salomo-Inseln) und Genyophrynidae (Genyophryne Thomsoni Blgr. auf der Südost-Insel).

Von den Pelobatidae erwähnt Boulenger im "Catalogue of the British Museum" 3 Arten (von. welchen eine unsichere) von Neu-Guinea. Es hat sich aber herausgestellt, dass zwei derselben nicht zu den Pelobatidae gehören (s. unten), die dritte aber, Batrachopsis (Lechriodus) melanopyga Dor., welche auch von den Aru-Inseln nachgewiesen ist, muss zu dieser Familie gerechnet werden. Zwar ist sie, die einzige ihres Genus, wie Fry ${ }^{1}$ ) hervorhebt, einigen Australischen Cystignathidae sehr ähnlich, die stark erweiterten Sacralfortsätze gestatten aber nicht sie bei dieser Familie einzuteilen, wenn auch zoogeographisch das Vorkommen einer Pelobatide auf Neu-Guinea, einer Familie, welche sonst auf die holarktische und die indische Region beschränkt ist und in Celebes und den Molukken fehlt, schwer zu erklären ist.

Die der Hauptsache nach Südamerikanisch-Australischen, im westlichen Teile des Indischen Archipels fehlenden Hylidae sind auf Neu-Guinea, welches ihnen durch seinen Reichtum an Wäldern ein günstiges Milieu bietet, in zahlreichen Arten anzutreffen. Sie gehören

1) Proc. R. Soc. Queensland, XXVII, 1915, p. 73. 
drei Genera, Hyla, Hylella und Nyctimantis, an. Die Gattung Hylella jedoch, welche sich nur durch das Fehlen der Vomerzähne von Hyla unterscheidet, ist sicher nicht natürlich. Ich habe sie mit Hyla vereinigt, umsomehr weil auch bei einigen Hyla-Arten in der Jugend die Vomerzähne noch fehlen.

Von der Gattung Nyctimantis, welche sonst nur noch von Süd-Amerika bekannt ist, kommen auf Neu-Guinea zwei Arten, N. granti Blgr. und papua Blgr., vor, beide nur im südlichen Teile der Insel.

Von Hyla sind eine grosse Zahl von Arten von Neu-Guinea beschrieben, welche aber wohl nicht alle aufrecht zu erhalten sind. So ist es sehr wahrscheinlich, dass einige derselben nur Jugendformen darstellen; welche bisweilen eine auffallende, aus hellen (im Leben wahrscheinlich immer gelben) Binden und Flecken zusammengesetzte Zeichnung aufweisen, die später verschwindet. Die papuanischen Arten, bei welchen solche Jugendformen bekannt sind, sind H. bicolor Gray, fallax Blgr. und impura Ptrs. et Dor. Junge H. fallax sind als Hylella Boulengeri Méh. beschrieben worden; H. Mácgregori Dgl.-Og. (thesaurensis Méh., nec Ptrs.) betrachte ich als junge $H$. impura, $H$. thesaurensis Ptrs. als junge $H$. macrops Blgr. (Salomo-Inseln). Auch H. albomaculata Vogt und papua v. Kamp. sind wohl auf junge Exemplare gegründet, vielleicht yon $H$. montana. Unvollständig beschrieben sind Hyla albolabris Wand., H. pulchra Wand. (= montana?), Hylella nigropunctata Meyer (= Hyla infrafrenata juv. ?) und Hylella Wolterstorffi Wern. (= Hyla arfakiana juv?).

Es bleiben dann die folgenden 26 Hyla-Arten von Neu-Guinea übrig: angiana Blgr.; arfakiana Ptrs. et Dor.; bicolor Gray; brachypus Wern.; caerulea White; chloronotus Blgr.; congenita Ptrs. et Dor.; eucnemis Lönnb.; fallax Blgr.; graminea Blgr.; humeralis Blgr.; impura Ptrs. et Dor.; infrafrenata Gthr. (=dolichopsis Cope); Jeudii Wern.; longicrus Blgr.; montana Ptrs. et Dor.; mystax v. Kamp.; nasuta Gray (= Semoni Bttgr.); obsoleta Lönnb.; papuensis Wern.; Pratti Blgr.; rhacophorus v. Kamp.; sanguineolenta v. Kamp. 1); Spengeli Blgr.; vagabunda Ptrs. et Dor.; Wollastoni Blgr. (vielleicht = arfakiana?).

Von der, wie die Hylidae hauptsächlich Südamerikanish-Australischen Familie der Cystignathidae beschrieb ich eine Art von Neu-Guinea unter dem Namen Phanerotis novae-guineae ${ }^{2}$ ). Nachher konnte Fry ${ }^{3}$ ) durch Untersuchung des Original-Exemplares des von Macleay ungenügend beschriebenen Ranaster convexiusculus konstatieren, dass diese Art, welche Boulenger mit einigem $\mathrm{Z}_{w e i f e l ~ \mathrm{zu}}$ den Pelobatidae gebracht hatte, mit Phanerotis novae-guineae synonym ist. Diese einzige von Neu-Guinea bekannte Cystignathide ist nur im dem südlichen Teile der Insel aufgefunden.

Die fast kosmopolitische, im grössten Teile von Australien jedoch fehlende Familie der Ranidae ist auf Neu-Guinea viel schwächer vertreten als im westlichen Teile des Indischen Archipels. Die Neu-Guinea-Arten gehören nur zwei Gattungen an: Platymantis und Rana.

Das frühere Genus Cornufer wurde von Boulenger ${ }^{4}$ ) nach dem Baue der Fingerscheiben wieder getrennt in Cornufer und Platymantis. Die Verbreitung von Cornufer wird aber dadurch eine discontinuirliche, indem es westlich (Burma, Borneo, Philippinen) und östlich (Salomound Fidschi-Inseln) von Neu-Guinea vorkommt, auf dieser Insel selbst aber zu fehlen scheint. C. unicolor Tsch. von Neu-Guinea wenigstens ist zweifelhaft: das von Tschudi erwähnte junge Exemplar aus dern Museum zu Leiden ist eine Sphenophryne cornuta.

Platymantis bewohnt die Philippinen, Halmahera, die Kei-Inseln, Neu-Guinea und benachbarte Inseln, den Bismarck-Archipel und die Salomo- und Fidschi-Inseln. P. corrugata A. Dum. ist, auch auf Neu-Guinea, die meist verbreitete Art, von welcher P. Moszkowskii Vogt nach der Beschreibung nicht spezifisch zu trennen ist. P. punctata Ptrs. et Dor. ist nur von dem westlichen Teile der Insel bekannt.

1) Boulenger (Zool. Jahrb., Suppl. XV, Bd. 1, 1912, p. 214) meint, dass diese Art mit $H$. infrafrencta zu vereinigen sei; sie scheint mir aber von dieser konstant zu unterscheiden durch die Gestalt der Schnauze und das Fehlen des hellen Unterlippenrandes.

2) Nova: Guinea, IX, livr. 1, 1909, p. 36 .

3) Mem. Queensland Mus., II, 1913, p. 47

4) Ann. Mag. Nat. Hist., (9) I, 1918, p. 372 
Die Rana-Arten von Neu-Guinea sind: arfaki Meyer, Daemeli Stdchn. (= novae-guineae v. Kamp.), grisea v. Kamp., grunniens Daud., macrodon Kuhl, macroscelis Blgr. (=waigeënsis v. Kamp.), novae-britanniae Wern., papua Less., Tornieri Vogt. Einige derselben sind aber unsicher. So scheinen mir macroscelis und Tornieri kaum von arfaki zu trennen; Daemeli, zugleich die einzige Ranide von Australien, ist sehr nahe verwandt, vielleicht synonym mit R. papua; grunniens ist nicht sicher verschieden von macrodon; novae-britanniae ist vielleicht dieselbe wie Krefftii Blgr. von den Salomo-Inseln, wenn nicht identisch mit papua ${ }^{1}$ ).

Am zahlreichsten sind auf Neu-Guinea die Engystomatidae vertreten, eine Familie, welche sonst in relativ wenigen Gattungen und Arten über Süd-Amerika, Afrika und in der Orientalischen Region, den gesamten Indischen Archipel mit einbegriffen, verbreitet ist. Erst vor kurzem hat Fry $^{2}$ ) auch einige wenige Arten, für welche er ein Genus Austrochaperina aufstellt, in Queensland nachgewiesen.

Die papuanischen Engystomatidae sind namentlich von v. Méhely in eine grosse Zahl von Gattungen eingeteilt worden, welche aber wohl sicher nicht alle berechtigt sind. So glaube ich Gnathophryne, Phrynixalus und Metopostira alle mit Hylophorbus (Mantophryne) vereinigen zu müssen, dessen Diagnose dann lautet: Pupille horizontal; Zunge gross, oval, hinten mehr oder weniger frei; Gaumen sahnlos, mit zwei Querfalten (Unterschied von Asterophrys, Copiula und Xenorhina), nur die hintere gezahnt (Unterschied von Choerophryne); Trommelfell sichtbar (Unterschied von Callula, Microhyla und Pomatops); Finger und Zehen frei (Unterschied von Cophixalus), die Spitzen mehr oder weniger erweitert.

Eine genaue Prûfung der Literatur und des mir zugänglichen Materiales hat mich weiter genötigt, die Gattungen Sphenophryne, Chaperina und Austrochaperina zu vereinigen, und Méhelyia, sowie einige der als zu Splienophryne gehörig beschriebenen Arten mit Oreephryne zusammenzubringen. Beide Gattungen, Sphenophryne und Oreophryne, sind dann gekennzeichnet durch das Vorhandensein von Procoracoid und Clavicula, durch den Besitz von einer oder zwei Gaumenfalten und durch erweiterte Finger- und Zehenspitzen. Bei Sphenophryne stösst die Clavicula an die Scapula, bei Oreophryne nicht. Der von v. Méhely ${ }^{3}$ ) betonte Unterschied in der Ausbildung des Procoracoid, nach welcher er seine Gattungen Oreophryne und Sphenophryne unterscheidet, halte ich für wenig wichtig. Die Vereinigung ron Chaperina mit Sphenophryne ist gegründet auf die wahrscheinlich richtige Annahme von Boulenger ${ }^{4}$ ), dass Chaperina ceratophthalmus v. Kamp. ein Synonym ist von Sphen. cornuta. Der Schultergürtel dieser typischen Art des Genus stimmt dann mit demjenigen von Méhely's Chaperina überein.

Auch Xenorhina und Verwandte bedürfen einer Revision. Im Jahre $18^{1 / 8} 8^{5}$ ) stellten Peters und Doria das Genus Xenobatrachus auf, mit der. Art ophiodon; es war namentlich durch den Besitz von zwei Gaumenzähnen hinter jeder Choane gekennzeichnet. V. Méhely ${ }^{6}$ ) bemerkt, nach der Beschreibung seines neuen Genus Choanacantha mit der Art rostrata: "This new genus is very closely allied to Peters and Doria's Xenobatrachus, but differs in the tongue, which is perfectly adberent behind; in the single toothlike bony prominence on each palatine bone; in the perfectly hidden tympanum; and in the separated outer metatarsals." Boulenger $\left.{ }^{7}\right)$ fügte diesem Genus eine neue Art, Ch. ${ }^{`}$ Méhelyi, hinzu.

Nachher erkannte v. Méhely ${ }^{8}$ ) die grosse Ähnlichkeit zwischen Choanacantha und Peters' Genus Xenorhina ${ }^{9}$ ), dessen einzige Art, $X$. oxycephalus Schleg. ("Bombinator oxycephalus") zwar keine Gaumenzähne hat, aber dennoch nicht generisch von Ch. rostrata getrennt wer-

1) Werners Angabe (Zool. Jahrb., Syst., XIII, 1900, p. 493), daśs $R$. novae-britanniae auch auf Sumatra vorkomme, beruht wohl auf einer Verwechslung mit irgend einer anderen Art, vielleicht $R$. nicobariensis.

2) Records Australian Mus., IX, No. 1, 1912, p. 87; Proc. R. Soc. of Queensland, XXVII, No. 4, 1915, p. 61.

3) Termész. Füz., XXIV, 1901, p.: $251 \mathrm{ff}$.

4) Trans. Zool. Soc. London, XX, Pt. 5, 1914, p. 251.

5) Ann. Mus. Genova, XIII, 1878, p. 432

6) Termész. Füz., XXI, 1898, p. 175.

7) Ann. Mus. Genova, (2) XVIII, 1897, p. 709.

8) Termész. Füz., XXIV, 1901, p. 231.

9) Monatsber. Akad. Berlin, 1863, p. 82 
den könnte. Das Genus Choanacantha wäre demnach zu streichen, während Ch. Méhelyi mit rostrata vereinigt und die inzwischen von Günther ${ }^{1}$ ) beschriebene Xenorhina atra als synonym mit $X$. oxycephalus betrachtet wurde.

Von diesem Genus Xenorhina beschrieb ich nachher einige neue Arten, von welchen $X$. macrops und ocellata ${ }^{2}$ ) in dem Besitze eines Zahnes (oder Stachels?) hinter jeder Choane mit $X$. rostrata übereinstimmen, während $X$. bidens ${ }^{3}$ ) und gigantea ${ }^{4}$ ) beiderseits zwei solche Zähne besitzen. Hierin ähneln sie also Xenobatrachus ophiodon und ich betrachte jetzt dieses Merkmal von genügender Wichtigkeit, um alle Arten mit Gaumenzähnen mit Xenobatrachus zu vereinigen. Zwar unterscheidet sich Xenobatrachus ophiodon auch durch die herzförmige, hinten freie Zunge von der ursprünglichen Diagnose von Choanacantha; eine teilweise freie Zunge fand ich aber auch by $X$. ocellata und der Herzform ist kein grosser Wert beizumessen. Hinsichtlich des 'Trommelfells ist nachgewiesen worden, dass dieses auch bei rostrata sichtbar ist, während Méhely's Angabe, dass bei dieser die.Metatarsalia getrennt sein sollten, wohl einem Irrtum zuzuschreiben ist.

Ich unterscheide also:

Xenobatrachus: 1-2 7ähne (Stachel ?) hinter jeder Choane.

Xenorhina: keine .Gaumenzähne.

Zu den Engystomatidae gehört auch Asterophrys turpicola S. Müll. Anfänglich von Schlegel ${ }^{5}$ ) als Ceratophrys turpicola S. Müll. beschrieben und mit Megalophrys montana verglichen, wurde diese Art von Boulenger ${ }^{6}$ ), der selbst keine Exemplare gesehen hatte, zu den Pelobatidae gebracht. Ich konnte nun im Naturhistorischen Museum zu Leiden zwei Exemplare, wahrscheinlich die Typen, untersuchen, welche mir zeigten, dass sie zu den Engystomatidae, in der Nähe von Xenorhina, zu bringen sind: der Schultergürtel gehört zum firmisternen Typus, der Mund ist zahnlos (auch die von Tschudi erwähnten Gaumenzähne fehlen), die Sacralfortsätze sind schwach erweitert; Procoracoid und Clavicula fehlen.

Ich komme so zu der nachfolgenden Liste der.Engystomatiden von Neu-Guinea: Asterophrys turpicola S. Müll.; Callulops Doriae Blgr.; Choerophryne proboscidea v. Kamp.; Cophixalus Geislerorum Bttgr. (= Oreophryne sp. ?); Copiula oxyrhina Blgr. (Phrynixalus oxyrhinus) ${ }^{7}$ ); Hylophorbus Biroi Méh., microtis Wern. (= Neuhaussi Vogt?), ocellatus Méh. (= Metopostira macra v: Kamp.), robustus Blgr., rufescens Macl. (= Mantophryne lateralis Blgr.); Liophryne brevipes Blgr., Kampeni Blgr., rhododactyla Blgr.; Oreophryne albopunctata v. Kamp., Anthonyi Blgr., ateles Blgr. (=verrucosa juv.?), Biroi Méh. (= Méhelyia lineata und affinis Wand.?), crucifera v. Kamp. (Cophixalus crucifer); Loriae Blgr., verrucosa Blgr.; Oxydactyla brevicrus v. Kamp.; Pomatops valvifera Barb. (= Hylophorbus sp.?); Sphenophryne basipalmata v. Kamp. $(=S$. punctata v. Kamp., Schlaginhaufeni Wand.?, quatuorlobata Wand.?), cornuta Ptrs. et Dor. (= Chaperina ceratophthalmus v. Kamp.), fusca Mocq., Klossi Blgr., macrorhyncha v. Kamp., polysticta Méh.; Xenobatrachus bidens v. Kamp., giganteus v. Kamp, macrops v. Kamp., ocellatus v. Kamp., ophiodon Ptrs. et Dor., rostratus Méh. (= Choanacantha Méhelyi Blgr.), Xenorhina oxycephalus Schleg. (= atra Gthr.),

Von den $\%(6$ Arten von Neu-Guinea (die zweifelhaften lasse ich ferner unberücksichtigt) gehören also zu den:

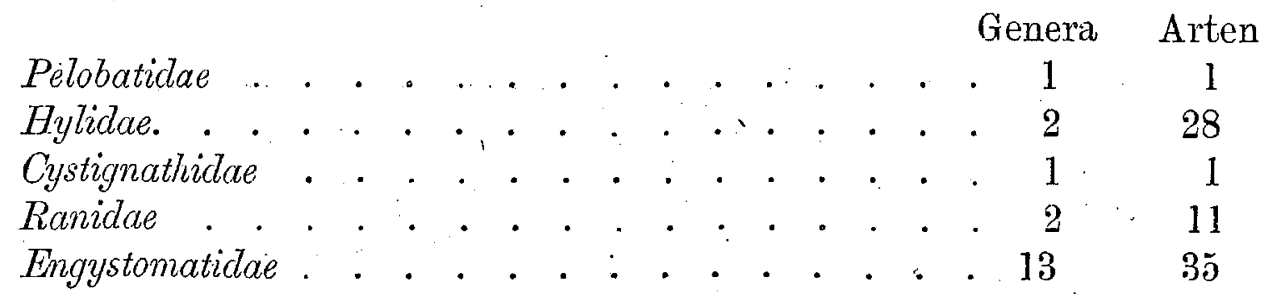

1) Novit. Zool, III, 1896, p. 184.

3) Nova Guinea, livr. 1, 1909, p. 39

2) Nova Guinea, IX, livr. 3, 1913, p. 460, 461

5) Abbild. neuer oder unvollständig bekannten Amphibien. Düsseldorf, 1837-44, p. 30.

6) Catalogue Batr. Sal., 1882, p. 443.

7) Copiula (?) rostelliferc Wand. ist ungenügend beschrieben und möglicherweise synonym mit Choerophryne proboscidea. 
Von diesen Arten sind bei weitem die meisten (5\%, also $\% 5 \%$ ) auf die Insel beschränkt, nur 19 sind weiter verbreitet, und zwar:

Batrachopsis melanopyga, Aru-Inseln.

Hyla bicolor, Aru-Inseln, Australien.

" brachypus, Bismarck-Arch.

” caerulea, Australien.

” congenita, Aru-Inseln.

" infrafrenata, Molukken, Timor, Kei-, Tenimber- und Arụ-Inseln, Misool, Waigeu, Salawati, Jobi, N.-Queensland.

" nasuta, Australien.

"vagabunda, Ceram.

Platymantis corrugata, Philippinen, Palau-Ins., Halmahera, Kei-Inseln, Batanta, Jobi, Bismarck-Arch., Salomo-Inseln, d'Entrecastaux-Inseln, St.-Aignan.

Rana arfaki, Waigeu.

" Daemeli, Australien.

"grunniens, Ambon, Saparua.

" macrodon, Hinter-Indien und fast der ganze Indische Archipel.

" macroscelis, Waigeu, Aru-Inseln.

"novae-britanniae, Bismarck-Arch.

” papua, Ceram, Aru- und Tenimber-Inseln, Batanta, Waigeu, Jobi, d'Entrecastaux-Inseln. Copiula oxyrhina, St.-Aignan.

Hylophorbus robustus, St.-Aignan.

Sphenophryne fusca, Borneo (?).

Aus den genannten Zahlen geht die grosse, schon früher ${ }^{1}$ ) von mir hervorgehobene Selbstständigkeit der Amphibienfauna von Neu-Guinea hervor.

Die Pelobatidae, Ranidae und Engystomatidae haben hauptsächlich westliche, die Hylidae und Cystignathidae südliche Verwandtschaftsbeziehungen. Die Amphibien deuten daher, wie andere Tiergruppen, auf die ehemalige Existenz von "Landbrücken" hin, welche Neu-Guinea einerseits mit den Molukken, anderseits mit Australien (und den Aru-Inseln) verknüpften. Von beiden Seiten sind Amphibien sowohl nach der Insel gelangt als von ihr aus weiter verbreitet.

Am ältesten und am leichtesten zu überschreiten muss die Verbindung mit Australien gewesen sein. Durch sie ist die Übereinstimmung zu erklären, welche existiert z.B. zwischen den Saügetieren, den Süsswasserfischen und unter den Amphibien den Hylidae von Australien und Neu-Guinea. Einige wenige von diesen Tieren (unter den Hylidae H. infrafrenata und vagabunda) haben sich später nach den Molukken ausbreiten können und zugleich hat Neu-Guinea längs demselben Wege die Indischen Bestandteile seiner Fauna, unter welchen die Ranidae und Engystomatidae, bekommen.

Einige Tatsachen der Amphibienverbreitung scheinen als Argumente dienen zu können für die Annahme von zwei "Molukkenbrücken", einer nördlichen nach Halmahera und einer südlichen nach Ceram. So scheinen Platymantis corrugata, sowie die Gattungen Hylophorbus und Oreophryne, welche alle von Halmahera bekannt sind, in den südlichen Molukken zu fehlen; auf die Ceram-Brücke deutet die Verbreitung von Rana grunniens, R. papua und Hyla vagabunda hin.

Von Neu-Guinea aus sind dann weiter einige Indische Amphibien-Arten in Australien eingewandert, nämlich Rana Daemeli und die vier von Fry beschriebenen Austrochaperina(Sphenophryne-)Arten. Wäre bei $R$. Daemeli vielleicht noch ein Transport über dem Meere möglich, für die Engystomatidae ist dieses nicht anzunehmen. Sie können dieselbe Landverbindung benützt haben, über welche früher die Hylidae nach Neu-Guinea gekommen waren,

1) Nova Guinea, v, livr. 1, 1906, p. 179 f. und IX, livr. 1, 1909, p. 45 ff. 
wahrscheinlicher ist es aber, dass die Verbindung zeitweilig unterbrochen gewesen ist und erst später durch eine neue, schmälere ersetzt. Es ist möglich, dass diesem Wege entlang auch $H$. infrafrenata, für welche Neu-Guinea das Zentrum der Verbreitung darstellt, nach Australien gelangt ist.

In der anderen Richtung ist diese jüngere Verbindung benutzt worden von einigen Arten, welche sich auf Neu-Guinea noch nicht weiter ausbreiten haben können als über die südliche Ebene. Es sind Hyla caerulea und nasuta, beide Arten, welche Neu-Guinea und Australien mit einander gemeinsam haben, und Ranaster convexiusculus, die einzige Cystignathide von Neu-Gruinea.

Die Amphibien, speziell die Anuren, sind durch ibren Formenreichtum und dadurch, dass ein Transport über den. Meere bei ibnen so gut wie ausgeschlossen ist, sehr geeignet in zoogeographischen Fragen Licht zu verschaffen. Demgegenüber steht der Nachteil, dass ihre Geschichte, durch fehlen paläontologischer Urkunden, so gut wie unbekannt ist. Auf das. Alter der Landverbindungen werfen sie daher kein Licht. 